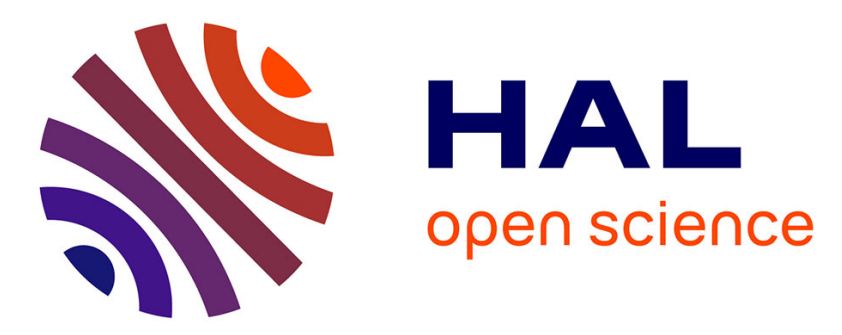

\title{
Emerson et William James, ou les vertus de la croyance Thomas Constantinesco
}

\section{To cite this version:}

Thomas Constantinesco. Emerson et William James, ou les vertus de la croyance . Revue Française d'Etudes Américaines, 2014, Transferts du religieux, 141, pp.56-68. 10.3917/rfea.141.0056 . hal01378890

\section{HAL Id: hal-01378890 \\ https://hal-univ-paris.archives-ouvertes.fr/hal-01378890}

Submitted on 24 Jan 2018

HAL is a multi-disciplinary open access archive for the deposit and dissemination of scientific research documents, whether they are published or not. The documents may come from teaching and research institutions in France or abroad, or from public or private research centers.
L'archive ouverte pluridisciplinaire HAL, est destinée au dépôt et à la diffusion de documents scientifiques de niveau recherche, publiés ou non, émanant des établissements d'enseignement et de recherche français ou étrangers, des laboratoires publics ou privés. 


\title{
EMERSON ET WILLIAM JAMES, OU LES VERTUS DE LA CROYANCE
}

\author{
Thomas Constantinesco
}

Université Paris Diderot

On a souvent dit d'Emerson qu'il était le premier philosophe américain avant William James, et les historiens de la philosophie américaine en quête de pères fondateurs ont eu tôt fait d'établir, entre le transcendantalisme de l'un et le pragmatisme de l'autre, une sorte de lignée, fût-elle paradoxale, qu'il s'agisse de retracer la continuité d'une pensée ou au contraire de fonder une contre-généalogie, de faire d'Emerson un proto-William James ou de James un anti-Emerson ${ }^{1}$. Pour Stanley Cavell, en revanche, une telle archéologie s'apparente toujours à une opération de refoulement (repression), car elle consiste au bout du compte à rabattre l'un sur l'autre, empêchant d'entendre la voix de chacun. À suivre Cavell, une telle enquête historiciste empêcherait surtout d'entendre celle d'Emerson, qu'il propose finalement d'inscrire dans la tradition du perfectionnisme, de Platon à Nietzsche, Heidegger et Wittgenstein, plutôt que dans celle du pragmatisme de John Dewey et William James (Cavell 215-223). Il n'en reste pas moins qu'Emerson et James semblent avoir eu en partage des objets de pensée communs, de sorte que c'est peut-être en commençant par confronter leurs textes que

\footnotetext{
${ }^{1}$ Dans American Philosophy and the Romantic Tradition, Russell Goodman situe Emerson, John Dewey et William James dans le sillage d'une même tradition romantique qui remonte à Coleridge. Si la filiation est d'abord conceptuelle, Goodman met en lumière les liens biographiques qui unissent Emerson et William James, dont le père, Henry Sr., partageait avec Emerson un même intérêt pour la théorie des correspondances de Swedenborg. Emerson était en outre un familier de la maison James et William prononcera, à l'occasion du centenaire de sa naissance en 1903, un éloge resté célèbre. Dans William James: Empirisme et Pragmatisme, David Lapoujade voit au contraire dans le pragmatisme de James une «rupture » paradoxale d'avec le transcendantalisme d'Emerson: «Certes, James en conserve certains aspects lorsqu'il invoque une confiance nécessaire dans le rapport avec soi, avec le monde ou avec les autres ; il n'en demeure pas moins que les coordonnées se sont modifiées. » (Lapoujade 1997, 120)
} 
l'on pourra le mieux percevoir la tonalité singulière de leur voix philosophique, si l'on admet avec Cavell qu'« en philosophie, c'est le son qui fait toute la différence » (216).

L'un de ces motifs partagés, c'est justement la question de la croyance, ou plutôt le rapport du sujet à ses croyances, qui s'envisage, dans les années 1840 comme dans les années 1890, sur fond de crise spirituelle et de désenchantement religieux. Le transcendantalisme, on le sait, naît d'un rejet de l'unitarisme qui domine à Boston dans les années 1830 et plus largement d'une défiance à l'égard des institutions religieuses, sociales et politiques, jugées inaptes à redonner à l'individu la place centrale qu'il a perdue dans le monde. Sans doute ce sentiment de confusion et de perte des repères s'est-il accentué à la fin du siècle, comme en témoigne par exemple L'Éducation de Henry Adams (1905) : dans cette autobiographie à la troisième personne, dont l'auteur est à la fois une connaissance d'Emerson et un ami des frères James, Henry Adams rêve ainsi que les sciences modernes, du darwinisme à la thermodynamique et la psychologie, puissent faire office de religions de substitution et permettent enfin de parer au chaos qui partout s'observe ; mais chaque fois se fait jour un peu plus que la seule vérité digne de foi, c'est la loi du désordre généralisé, qui rend d'autant plus urgente la nécessité de se donner de nouvelles idoles (Imbert 2008 et 2012). Au fil du XIXe siècle, le sujet est décentré, relégué aux marges d'un univers qui lui-même se disloque, et le besoin de croire se renforce à mesure que s'étiole la confiance en soi et dans le monde. Il est dès lors remarquable d'observer, d'Emerson à James, une sorte de relation en chiasme autour de la croyance et de ses vertus, notamment pratiques : chez Emerson, la crise de la croyance croit pouvoir se résoudre dans la foi en un sujet tout-puissant, tandis que chez William James, il semble au contraire que ce soit la crise du sujet qui trouve son issue dans l'affirmation renouvelée de la volonté de croire. Mais si l'individualisme émersonien repose sur une foi inconditionnelle en la divinité du moi, ce «moi » se révèle 
toutefois instable et divisé et prend la forme d'un complexe de pensées et d'humeurs contradictoires auxquelles le sujet, qui en est l'effet, ne peut finalement pas se fier. James, à l'inverse, fait d'emblée le constat de la division du sujet, qu'il conçoit, non plus comme un substrat stable aux contours bien délimités, mais comme un ensemble de coordonnées fluctuantes évoluant dans un système de croyances collectives et de confiance mutuelle. L'économie de la confiance qui en découle exige cependant que « quelqu'un », « quelque part», en garantisse la stabilité, ce qui revient à réintroduire subrepticement l'idée d'un sujet un et indivisible dont James avait pourtant mis au jour la fêlure dès les Principles of Psychology en 1890.

D'une philosophie à l'autre, c'est cette relation en miroir autour de la constitution/destitution du sujet et de ses croyances - du sujet par ses croyances - que je souhaiterais suivre ici en rapprochant quelques passages représentatifs de l'une et de l'autre. Il s'agira de mettre en lumière la façon dont les deux pensées s'organisent selon une logique partagée, sans toutefois présupposer que le signifiant « belief » renvoie à un même concept dans les deux œuvres, ni qu'il reste identique à lui-même chez Emerson et chez James, l'un et l'autre reconfigurant sans cesse le sens de la croyance au fil de reprises lexicales qui masquent parfois des ruptures conceptuelles. Accompagner le mouvement de la pensée à travers des contextes distincts, un vocabulaire fuyant et des significations de fait labiles devrait permettre, c'est du moins l'une des hypothèses de cette étude, de mieux faire apparaître ce qu'Emerson et James ont en commun, mais aussi, et par contrecoup, ce qui les distingue, à commencer peut-être par l'horizon conceptuel dans lequel ils s'inscrivent, Emerson l'ancien pasteur continuant de proposer ce que l'on pourrait appeler un dispositif théologique où la croyance est toujours croyance en une forme de divin, là où James tient le fait religieux à plus grande distance 
et découple la question de la croyance de celle de la foi pour la penser selon des coordonnées à la fois psychologiques et pragmatiques.

\section{« Trust Thyself»}

Dans une conférence intitulée «Lecture on the Times » (1841), Emerson offre une vue télescopique de l'histoire nationale et compare l'esprit de ses contemporains à celui des générations antérieures. Il conclut sans ambages : « our torment is Unbelief» $(165)^{2}$. Dix ans plus tard, en revanche, dans un essai paradoxalement consacré à Montaigne et au scepticisme, il balaie ce constat et affirme : « we are natural believers » (701). Oscillant entre la vision désenchantée d'un monde sans Dieu et un besoin compulsif, voir atavique, de croire, Emerson tient constamment un double langage, «now sceptical [...] and now religious », pour reprendre les catégories de «Experience » (484). À n'en pas douter, cette hésitation renvoie à son propre goût pour la contradiction, mais elle peut également se lire comme une manifestation de ce qu'il appelle, bien avant W. E. B. Du Bois, sa « double conscience» (this double consciousness [« The Transcendentalist» 205]) ou, dans le vocabulaire clinique de William James, comme le symptôme d'un « moi divisé » (divided self).

Tout commence donc avec la prise de conscience que l'Amérique du premier XIXe siècle est saisie par une crise spirituelle qui conduit à un insupportable immobilisme, conséquence d'un scepticisme de mauvais aloi : «A new disease has fallen on the life of man. [...] [Our] torment is Unbelief, the Uncertainty as to what we ought to do; the distrust of the value of what we do » («Lecture on the Times »165). Ou bien, dans les mots de "The American Scholar», dès 1837: "Yes, we are the cowed,-we the trustless. » (65) La pathologie de l'incroyance se double d'autres maladies : l'incertitude,

\footnotetext{
${ }^{2}$ Sauf mention contraire, les textes d'Emerson sont cités dans l'édition de Joel Porte dont la référence figure en en fin d'article.
} 
la peur et l'inaction. Toutefois, à renverser la polarité, cela signifie du même coup que la croyance aurait à voir avec l'action, mais aussi avec la question de la "valeur », et ce maillage conceptuel n'est pas sans rapport avec celui que tisse William James, pour qui la croyance se comprend aussi comme le moteur de l'action (willingness to act [James 1992, 458]) et la mesure de sa valeur pratique (its cash-value in experiential terms [James 1998, 572]).

Si l'incroyance est une pathologie sociale, elle s'éprouve aussi au niveau individuel, ce dont Emerson a lui-même fait l'expérience : après le décès de sa première épouse Ellen en 1831, il traverse une crise de conscience qui le conduit l'année suivante à démissionner de sa charge de pasteur de la Seconde Église de Boston et la raison qu'il invoque tient précisément à ce qu'il ne croit plus aux rites de l’Eglise, en particulier à celui de la communion qu'il voit comme une « forme » vide, dénuée de signification. Le christianisme n'est plus pour lui une religion vivante, mais une institution morte (a dead church [«Self-Reliance» 263]) dont les articles de foi ne sont plus respectés qu'à force d'habitude. Dans son sermon de la démission, "The Lord's Supper », il entreprend de montrer que l'eucharistie est devenue un rituel sclérosé et dépourvu de sens auquel il lui est impossible d'accorder le moindre crédit : « My friends, the kingdom of God is not meat and drink. Forms are as essential as bodies. It would be foolish to declaim against them, but to adhere to one form the moment it is outgrown is foolish. That form only is good and Christian which answers its end. » (Emerson 2001, 24) La croyance est ici affaire d'adhésion, de coïncidence, mais aussi d'efficace et d'utilité. Une croyance est vraie, elle est fondée, parce qu'elle répond à sa fonction, parce qu'elle fonctionne - parce que « ça marche » dirait William James dans un registre plus démotique qui caractérise à bien des égards son style philosophique : «because it works » (James 1998, 576). Chez Emerson, la croyance paraît relever d'une pragmatique avant l'heure et sa validité se 
mesure à l'aune de son efficacité dans une situation donnée : elle n'est jamais fixée pour de bon, elle est toujours variable et relative, fonction de sa pertinence pour moi : «fluxional, [...] vehicular and transitive », pour reprendre les termes de " The Poet» (463). Dans le cas contraire, ce n'est plus une croyance, mais une forme d'idolâtrie dont le respect aveugle entraîne la paralysie.

Il semble néanmoins que les contemporains d'Emerson aient oublié cette leçon et qu'ils soient devenus la proie de l'idole de leur époque qui a pour nom le conformisme ou la conformité. À en croire Emerson, ses concitoyens ne se contentent pas de se conformer à l'opinion majoritaire comme l'avait diagnostiqué Tocqueville, d'imiter les autres plutôt que d'agir par eux-mêmes, mais ils se conforment également à la loi de leurs propres actions passées et imitent des versions antérieures et dépassées d'euxmêmes, au lieu de se fier à ce qu'ils sont et deviennent à chaque instant : "The other terror that scares us from self-trust is our consistency; a reverence for our past act or word, because the eyes of others have no other data for computing our orbit than our past acts, and we are loath to disappoint them.» («Self-Reliance» 256) Emerson déplore que ses contemporains n'aient plus confiance en eux-mêmes et cèdent à la tyrannie de l'habitude qui les pétrifie et les contraint à la répétition. Cette condamnation du grégarisme permet toutefois l'amorce d'un mouvement de reprise (self-recovery [«Circles» 407]). Refusant d'accréditer sa propre incroyance, littéralement incrédule devant sa foi vacillante, le sujet émersonien s'efforce de reprendre le contrôle de luimême pour mieux recommencer à croire en ses possibilités. L'accent est alors mis sur l'autre pôle qu'évoque «Experience», celui du sentiment religieux. Pour citer une nouvelle fois l'essai sur Montaigne : «We suddenly believe in will » (704). Pourtant, si cette volonté émane du sujet, celui-ci semble moins en être l'origine ou le maître que l'effet: "So in accepting the leading of the sentiments, it is not what we believe 
concerning the immortality of the soul, or the like, but the universal impulse to believe, that is the material circumstance, and is the principal fact in the history of the globe. » («Experience » 486, italiques dans l'original) En insistant ainsi sur le rôle du sentiment et en rapportant l'origine de nos actions à une pulsion de croyance, Emerson paraît de nouveau anticiper ce que William James dira, quoique sur un ton moins prophétique, de la manière dont les idées que nous tenons pour vraies, celles auxquelles nous croyons, nous guident dans l'existence :

[True ideas] lead us, namely, through the acts and other ideas which they instigate, into or up to, or towards, other parts of experience with which we feel all the while [...] that the original ideas remain in agreement. [...] The function of agreeable leading is what we mean by an idea's verification. [...] [The] truth of a state of mind means this function of a leading that is worth while.

(James 1998, 574-575, l'auteur souligne)

Pour Emerson comme pour James, la conduite de la vie (leading) est affaire de croyance. On ne sera dès lors pas surpris de trouver sous la plume d'Emerson en 1860, dans un essai intitulé «Worship » et justement publié dans The Conduct of Life, ces deux aphorismes: «We are born believing. A man bears belief, as a tree bears apples. » (1056)

Or, ce en quoi le sujet croit, c'est d'abord en lui-même et en sa propre divinité. Comme le rappelle Emerson dans «Experience » : «We believe in ourselves, as we do not believe in others. » (488) L'individualisme devient alors la nouvelle religion des temps modernes et, dans le credo transcendantaliste, la créature prend la place de son Créateur pour devenir l'objet d'un nouveau culte : «Trust thyself: every heart vibrates to that iron string.» («Self-Reliance» 260). Paradoxalement, le nouvel article de foi ne vient pas tant contredire le plan divin qu'il ne le confirme, car Emerson prône la 
correspondance de l'ordre providentiel et de la volonté individuelle : se faire confiance, avoir foi en soi-même, est en réalité le moyen le plus sûr de retrouver Dieu en soi («God is here within » [272]), et ainsi de recomposer une totalité harmonieuse et de participer à l'avènement d'une communauté de divins égaux. Ce mouvement d'intériorisation de la transcendance s'énonce dès les premières lignes de "Self-Reliance », dans un passage bien connu aux accents millénaristes :

To believe your own thought, to believe that what is true for you in your private heart is true for all men,-that is genius. Speak your latent conviction, and it shall become the universal sense; for the inmost in due time becomes the outmost,- and our first thought is rendered back to us by the trumpets of the Last Judgment.

(«Self-Reliance », 259)

Ce renversement apocalyptique aux allures de forçage, où s'observe également le travail d'invention philosophique de l'écriture d'Emerson, ouvre la voie à l'avènement d'un nouvel âge de gloire qui serait aussi un nouvel âge de la croyance. Comme l'indiquera « Worship » quelque vingt ans plus tard : «All the great ages have been ages of belief. » (1063) Et conformément au lien qui unit la croyance et l'action, la foi renouvelée dans l'individu permet de mettre un terme à la paralysie générale qui afflige la nation : «But the believer not only beholds his heaven to be possible, but already to begin to exist » («Man the Reformer»147). À l'instar de James, pour qui nos croyances sont d'abord tournées vers le monde d'ici-bas, Emerson suggère que c'est par ses croyances que le sujet se donne un monde. Cette dernière citation vient d'ailleurs au terme d'un passage où Emerson commence par dénoncer les errements d'une génération qui a perdu la foi («I see at once how paltry is all this generation of unbelievers » [147]) avant d'énoncer ce qui s'apparente à une prophétie auto-réalisatrice par où le sujet et l'Amérique reprennent le contrôle d'eux-mêmes pour mettre en œuvre leur destinée commune. À 
relire ce paragraphe, on s'aperçoit toutefois que si l'Amérique s'est détournée de Dieu, c'est pour lui substituer le fétichisme de la monnaie: «The Americans have many virtues but they have not Faith and Hope. I know no two words whose meaning is more lost sight of. [...] The Americans have no faith. They rely on the power of a dollar; they are deaf to a sentiment. » (146-147) Le véritable rival du christianisme ne serait donc pas tant le scepticisme que le capitalisme, de sorte que l'enjeu consiste moins pour le sujet à sortir de l'incroyance et retrouver la foi, qu'à se libérer de la domination de croyances qui ne lui viennent pas de lui-même mais s'imposent à lui de l'extérieur. C'est à cette seule condition qu'un sujet libre peut advenir et que l'ordre du monde peut être restauré, ainsi que le résume une phrase de « New England Reformers » (1844) dont le rythme ternaire, si fréquent dans la rhétorique émersonienne, vient asseoir la prophétie et lui donne l'assurance de la vérité : «Men believe that the best is true; that right is done at last; or chaos would come. » (607-608)

\section{Croyance, confiance, crédit}

Cette dernière citation rassemble une série de motifs que l'on retrouve chez William James, qui associe lui aussi la croyance à la vérité, à l'action et à une forme de mise en ordre du monde. Même si l'enjeu est plus pragmatique que moral, James conçoit également la volonté de croire comme une réponse possible à ce qu'il perçoit comme une crise de l'action dans un monde qui a cessé de faire sens. On sait que « The Will to Believe », conférence prononcée en 1896 devant un public composé d'étudiants en philosophie de Yale et Brown et publiée l'année suivante, est en partie une réponse à deux autres textes publiés vingt ans auparavant : d'un côté, «The Fixation of Belief » de C. S. Peirce, à qui est dédié l'ouvrage dans lequel figure ce texte de James, et de l'autre, «The Ethics of Belief» du philosophe anglais William K. Clifford, que James réfute 
explicitement. Le premier de ces deux textes s'interroge sur l'épistémologie de la croyance et sa méthode : selon quelles procédures et à quelles conditions croyons-nous ce que nous croyons? Le second de son côté déplace la question sur le terrain de la morale et assimile croyance juste et croyance vraie pour affirmer : « it is wrong always, everywhere, and for anyone, to believe anything upon insufficient evidence » [Clifford 295]). Pour James en revanche, l'essentiel ne tient pas aux justifications a priori de nos croyances, qu'elles soient logiques ou morales, mais à leurs justifications pratiques ou pragmatiques a posteriori, c'est-à-dire à la manière dont nos croyances nous ont poussés à agir. Ce débat autour des différentes formes de justification de la croyance peut expliquer pourquoi James inscrit «The Will to Believe», non sans humour, dans la tradition homilétique et présente son texte comme « un sermon sur la justification par la foi », ou plutôt comme « un essai pour justifier de la foi » (« a sermon on justification by faith [...] an essay in justification of faith » [James 1992, 457]). Or, ce qui valide une croyance et la justifie, c'est le résultat de l'action qu'elle aura engendrée et qui nous aura fait accepter de courir les risques qu'elle impliquait. C'est d'ailleurs là tout le problème : nous n'agissons pas ou plus par peur des risques, par crainte des conséquences pour nous-mêmes. Car, comme le rappelle James dans « The Sentiment of Rationality » : «In the total game of life we stake our persons all the while » (527). En d'autres termes, la vie est une aventure dangereuse et pour la mener à bien, il faut croire fermement à la possibilité du succès, c'est-à-dire accorder une confiance suffisante d'abord à nousmêmes, mais aussi aux autres et au monde qui nous entoure. Tout est donc affaire de confiance et il n'est pas étonnant que « The Will to Believe » mobilise la métaphore du jeu, et même son langage parfois cru et direct («the language of the gaming-table » [460]), ni que James y invoque simultanément le célèbre pari de Pascal à l'appui de sa démonstration (460). Nous appartenons ainsi à une communauté de croyants et de 
parieurs dans laquelle nous plaçons notre confiance afin d'assurer notre mise : «our faith is faith in someone else's faith [...]. Our belief in truth itself, for instance, that there is a truth, that our minds and it are made for each other-what is it but a passionate affirmation of desire, in which our social system backs us up? » (463)

Le réseau de croyances enchâssées dans lequel nous sommes pris et grâce auquel nous menons nos existences ne tient que parce qu'il repose sur la confiance mutuelle de participants mus par un même désir de croire, et la philosophie pragmatique de James prend ici les allures d'un roman sentimental dont les personnages s'abandonnent à leur passion commune de la vérité, apparemment protégés de tout revers de fortune par la main invisible du « système social ». Ce faisant toutefois, la vérité elle-même cesse d'être une valeur refuge, un étalon inaltérable, car elle dépend du crédit que nous lui accordons et fluctue au gré des variations que subit son cours sur le marché de la confiance :

Truth lives, in fact, for the most part on a credit system. Our thoughts and beliefs "pass," so long as nothing challenges them, just as bank-notes pass so long as nobody refuses them. But this all points to direct face-to-face verifications somewhere, without which the fabric of truth collapses like a financial system with no cash-basis whatever. You accept my verification of one thing, I yours of another. We trade on each other's truth. But beliefs verified concretely by somebody are the posts of the whole superstructure.

(James 1998, 576-577)

Loin de s'exclure mutuellement, la vérité et la confiance, la certitude et la croyance, se soutiennent et se renforcent les unes les autres. En un sens, Emerson ne disait pas autre chose dans son essai « Character » (1844) lorsqu'il déclarait : «It is the privilege of truth to make itself believed. » (498) Si Kant affirmait avoir dû « supprimer le savoir pour lui substituer la croyance » afin d'établir un partage strict entre l'usage théorique et l'usage 
pratique de la raison (Kant 49), William James comme Emerson suggèrent au contraire que l'un est fonction de l'autre, et vice-versa: «la vérité et la croyance, bien que distinctes, dépendent l'une de l'autre et s'entrecroisent : tel un mécanisme à deux temps, l'une est la condition de l'autre. » (Imbert 2013, 14) Mais en même temps, on voit bien qu'au lieu de garantir la stabilité de la « superstructure », croire en la vérité ouvre la voie à tous les abus de confiance possibles. À faire commerce de la vérité, le risque est grand que les gages de certitude ainsi échangés se muent en monnaie de singe, en vertu de la loi de Gresham selon laquelle la mauvaise monnaie chasse toujours la bonne. Le transfert de truth à trust, sur fond d'étymologie partagée, autorise escroqueries et arnaques en tous genres (Imbert 2013,13). C'est précisément parce que la vérité est affaire de foi que l'on ne peut s'y fier. Il suffit pour s'en convaincre de songer au dernier roman publié par Melville de son vivant, The Confidence Man (1857), qui met en scène les tribulations de la vérité et les fluctuations de la foi à bord d'un vapeur ironiquement baptisé le Fidèle où tous les passagers sont finalement des escrocs en puissance (Imbert 2013, 8-23 ; Lapoujade 2010, 1218-25).

Ce scénario apocalyptique que déploie Melville et que James évoque sur le mode du déni, ce scénario où le système de la vérité finirait par s'effondrer («the fabric of truth collapses »), Emerson en donnait déjà les grandes lignes dans Nature en 1836. Le chapitre consacré au langage laisse en effet entrevoir la possibilité que nous vivions dans un monde de contrefaçons peuplé d'escrocs et de mystificateurs cherchant à abuser de notre confiance pour mieux nous détrousser: « when [...] duplicity and falsehood take the place of simplicity of truth, the power over nature as an interpreter of the will, is in a degree lost; new imagery ceases to be created, and old words are perverted to stand for things which are not; a paper currency is employed, when there is no bullion in the vaults. » (Nature 22) Mais pour Emerson comme pour James, à la 
différence de Melville, la menace n'est jamais envisagée que comme une limite épistémologique, car il existerait un étalon universel en réserve, capable de garantir la véracité de nos croyances : «But wise men pierce this rotten diction and fasten words again to visible things; so that picturesque language is at once a commanding certificate that he who employs it, is a man in alliance with truth and God» (Nature 23) ; "But beliefs verified concretely by somebody are the posts of the whole superstructure » (James 1998, 577). Revenant sur cette idée, James reconnaît que l'on ne peut se contenter de postuler que nos croyances sont vérifiables. Il faut, à un moment donné, qu'elles soient effectivement vérifiées : «The untrammelled flowing of the leadingprocess, its general freedom from clash and contradiction, passes for its indirect verification; but all roads lead to Rome, and in the end and eventually, all true processes must lead to the face of directly verifying sensible experiences somewhere »(581). « Tous les chemins mènent à Rome », de sorte que «quelqu'un », « quelque part », dans cette économie de la confiance qui ressemble aussi à une philosophie du langage ordinaire appliquée, doit assurer la vérité de nos croyances en apportant la preuve de la validité des siennes. Chez Emerson, cette tâche est confiée aux « hommes sages » (wise men), ces poètes qui sont « en harmonie avec la vérité et avec Dieu ». Chez James, il s'agit d'un anonyme (somebody). Mais le résultat est le même: la volonté de croire, tout comme la confiance en soi, exigent d'au moins un individu qu'il demeure identique à luimême dans ses convictions, c'est-à-dire qu'il croie à l'identité de sa volonté et l'unité de sa conscience. Au bout du compte, la « volonté de croire » impliquerait donc de poser un sujet un et indivisible.

\section{Flux de conscience}


La philosophie de William James est cependant tout sauf une philosophie moniste. Elle est foncièrement pluraliste (Madelrieux 317-348) et envisage la conscience comme une série de flux aux directions parfois contraires. C'est notamment l'une des leçons des Principles of Psychology (1890) où James établit, au chapitre X intitulé «The Consciousness of Self », une distinction entre l'ego, à savoir l'idée que je me fais de moimême, et le moi «empirique» ou social, qui existe par la reconnaissance d'autrui. D’entrée de jeu, le sujet est divisé d'avec lui-même et ces divisions ne cessent de se creuser : non seulement le moi empirique est, par nature, multiple, puisqu'il dépend de la perception que les autres ont de moi, mais il en va de même pour l'ego, toujours en proie à la scission interne, tiraillé qu'il est entre des pensées et des élans contradictoires. Alors qu'Emerson célébrait l'individu et en faisait le siège d'un divin intériorisé, James assimile, dans une métaphore à la fois irrévérencieuse et révélatrice de son attrait pour les images communes, les différents états du moi à du bétail livré à lui-même dans les prairies de l'Ouest («a herd of cattle let loose for the winter on some wide western prairie » [James 1981, 317]), attendant qu'un ou des gardiens viennent momentanément rassembler le troupeau. Ce faisant, James décrit un sujet qui, s'il aspire à l'unité, fait à chaque instant le constat de sa désunion : il n'y a donc pas de «sujet» au sens d'une entité stable et identique à elle-même, mais des états de conscience en flux (Bordogna 514-515).

Ceci soulève toutefois une redoutable difficulté : comment élaborer à la fois une économie de la confiance et une psychologie de la croyance en l'absence de sujet de l'une et de l'autre? David Lapoujade a montré que « ce qui est premier [chez James], ce sont les interprétations, les croyances: non pas un «je crois», mais une intensité, une émotion qui nous traverse et nous fait croire. Ce n'est pas un sujet qui fait les interprétations, mais l'inverse : le sujet se fait dans les interprétations ; mieux, il est lui- 
même une interprétation.» (Lapoujade 1997, 39) Le sujet n'est pas l'auteur de ses croyances, mais leur effet, et ce qui se dessine à l'horizon de cette psychologie de la croyance paradoxale, c'est une communauté de la foi sans fondement ouvrant sur un monde de signes que l'on ne peut qu'interpréter, mais jamais vérifier, sinon après-coup dans une forme de validation rétrospective.

À bien des égards, toutefois, il n’y a pas non plus chez Emerson de sujet de la confiance en soi, comme le suggère cette question faussement rhétorique que pose «Self-Reliance » : « Who is the Trustee? What is the aboriginal Self, on which a universal reliance may be grounded? » (268) En guise de réponse, Emerson décrit un sujet passif, agi de l'intérieur par des flux d'énergie qui circulent en lui et le façonnent : «We lie in the lap of immense intelligence, which makes us receivers of its truth and organs of its activity. When we discern justice, when we discern truth, we do nothing of ourselves, but allow a passage to its beams. » (269) Comme chez James, le sujet est un «nom de position» («a noun of position» [James 1912, 170]), une convention, et non une substance. Et sa position, justement, fluctue constamment, au gré des humeurs contradictoires qui le traversent et le transportent : « our moods do not believe in each other» («Circles» 406); «I am always insincere, as always knowing there are other moods» («Nominalist and Realist» 587). Si « Experience» nous apprend que notre confiance dans les autres est fort mal placée (488), ces deux essais suggèrent de leur côté que nous ne pouvons pas davantage croire en nous-mêmes.

Dans la perspective transcendantaliste cependant, ce constat désolé n'est jamais que le prélude à la reconquête du sujet par lui-même. La prise de conscience de la décomposition du sujet annonce son inéluctable recomposition. Cette logique, selon laquelle la division du sujet ne serait qu'un état transitoire, est précisément celle que William James met au jour au chapitre VIII de The Varieties of Religious Experience 
(1902), intitulé « The Divided Self and the Process of its Unification ». Il s'intéresse aux crises spirituelles de quelques grands mystiques, de Saint Augustin à Bunyan et Tolstoï, et décrit la « discordance » entre le sujet et lui-même, son « hétérogénéité intérieure » (James 1902, 156, 168), avant de présenter la volonté de croire comme un principe organisateur capable de pallier ces divisions internes : «Now in all of us, however constituted [...], does the normal evolution of character chiefly consist in the straightening out and unifying of the inner self. The higher and the lower feelings, the useful and the erring impulses, begin by a comparative chaos within us - they must end by forming a stable system of functions in right subordination. » (158) Quelques pages plus loin, James poursuit dans la même veine: «the process of remedying inner incompleteness and reducing inner discord is a general psychological process, which may take place with any sort of material, and need not necessarily assume the religious form » (163). Conformément au sous-titre de l'ouvrage, «A Study in Human Nature », le pathologique n'est donc qu'une manifestation exacerbée de l'état normal du psychisme et la résolution des tensions, une pente naturelle. De ce point de vue, la folie serait l'autre nom de la foi et la certitude de la révélation - qui soutient ces mystiques érigés par James en cas d'école, en figures représentatives de tout un chacun - apparaît comme une manière parmi d'autres de réunir des pulsions contraires. Mais en un sens, ceci n'est au fond que la reprise de ce que suggérait déjà Emerson dans « Experience »:

So is it with us, now skeptical, or without unity, because immersed in forms and effects all seeming to be of equal yet hostile value, and now religious, whilst in the reception of spiritual law. Bear with these distractions, with this coetaneous growth of the parts: they will one day be members, and obey one will. On that one will, on that secret cause, they nail our attention and hope. Life is hereby melted into an expectation or a religion. 
Chez l'un comme chez l'autre, la volonté de croire s'apparenterait donc en définitive à une fiction salutaire que se donne le sujet pour faire barrage à la menace de son effondrement.

$\mathrm{Au}$ bout du compte cependant, si la logique conceptuelle semble, au moins en partie, analogue, les voix philosophiques dans lesquelles elle s'énonce restent distinctes, ce qui permet peut-être de mesurer l'écart entre ces deux philosophies : illustrant une nouvelle fois le «ton apocalyptique» d'Emerson, pour parler avec Derrida, cette dernière citation signe l'horizon eschatologique du transcendantalisme, où la philosophie a encore partie liée avec la religion, tandis que les images volontairement communes de William James et son recours manifeste à la langue ordinaire sonnent l'avènement d'une nouvelle manière de faire de la philosophie à l'ère de la mort de Dieu, «philosophie populaire», comme l'indique le sous-titre de The Will to Believe, où ce qui unit la communauté n'est plus tant la croyance en la possibilité d'un autre monde à venir que la nécessaire confiance dans ce monde que nous faisons.

\section{OUVRAGES CITÉS}

BoRDoGna, Francesca. «Inner Divisions and Uncertain Contours: William James and the Politics of the Modern Self ». The British Journal for the History of Science 40, $\mathrm{n}^{\circ} 4$ (2007): 505-536.

CAVELL, Stanley. "What's the Use of Calling Emerson a Pragmatist? ». Emerson's Transcendental Etudes, Ed. David J. Hodge. Stanford: Stanford UP, 2003. 215-223.

Clifford, William K. « The Ethics of Belief ». Contemporary Review 29 (1876-1877): 290309. 
DeRRIDA, Jacques. D'un ton apocalyptique adopté naguère en philosophie. Paris : Galilée, 1983.

EmERSON, Ralph Waldo. Essays and Lectures. Ed. Joel Porte. New York: The Library of America, 1983.

-. Emerson's Prose and Poetry. Ed. Joel Porte and Saundra Morris. New York: Norton Critical Edition, 2000.

Goodman, Russell. American Philosophy and the Romantic Tradition. Cambridge: Cambridge UP, 1990.

IMBERT, Michel. «L'heure de vérité dans The Confidence-Man de Melville ». RFEA 133 (2013) : 8-24.

-. "Henry Adams and The Grammar of Sciences ». Science and American Literature in the 20th and 21st Centuries. From Henry Adams to John Adams. Ed. Claire Maniez et al. Newcastle-upon-Tyne: Cambridge Scholars Publishing, 2012. 3-19.

—. «The Education of Henry Adams ou la chronique du chaos ». RFEA 118 (2008): 111125.

JAMES, William. The Principles of Psychology: The Works of William James. Ed. Frederick H. Burkhardt. Cambridge: Harvard UP, 1981.

-. The Will to Believe and Other Essays in Popular Philosophy (1897). William James: Writings 1878-1899. Ed. Gerald F. Myers. New York: The Library of America, 1992.

-. The Varieties of Religious Experience: A Study in Human Nature (1902) et Pragmatism (1907). William James: Writings 1902-1910. Ed. Bruce Kuklick. New York: The Library of America, 1998. 1-477 et 479-623.

-. Essays in Radical Empiricism. New York : Longmans, Green, and co, 1912.

KanT, Emmanuel. Critique de la raison pure. Paris: GF-Flammarion, 1987.

LAPOUJADE, David. William James: Empirisme et Pragmatisme. Paris: PUF, 1997. 
-. «L'escroc à la confiance ou l'efficace du faux ». Herman Melville. Bartleby, Billy Budd et autres romans. Éd. Philippe Jaworski et al. Paris : Gallimard, Bibliothèque de la Pléiade, 2010. 1214-1231.

MADELRIEUX, Stéphane. William James: l'attitude empiriste. Paris : PUF, 2008.

PeIRcE, Charles Sanders. «The Fixation of Belief » (1877). The Essential Peirce, vol. 1. Ed. Nathan Houser et Christian Kloesel. Bloomington: Indiana UP, 1992. 109-123. 